\title{
Ferroplasmons: Novel Plasmons in Metal-Ferromagnetic Bimetallic Nanostructures
}

J.Ge ${ }^{1}$, A. Malasi ${ }^{2}$, N. Passarelli ${ }^{3}$, L. A. Pérez ${ }^{3}$, E. A. Coronado ${ }^{3}$, ,R. Sachan ${ }^{1,4}$, G. Duscher ${ }^{1,4}$ and R. Kalyanaraman ${ }^{1,2}$

${ }^{1}$ Department of Material Science and Engineering, University of Tennessee, Knoxville, Tennessee 37996, USA

2Department of Chemical and Biomolecular Engineering, University of Tennessee, Knoxville, Tennessee, 37996, USA

3INFIQC, Departamento de Fisicoquímica, Facultad de Ciencias Químicas, Departamento de Fisicoquímica, Universidad Nacional de Córdoba, Córdoba 5000, Argentina

${ }^{4}$ Material Science and Technology Division, Oak Ridge National Laboratory, Oak Ridge, TN 37831, USA

New plasmonic materials are required to enable potential strong coupling of light and magnetism, which could lead to improved data storage and/or completely novel optical materials [1, 2]. Bimetallic materials are a promising new way to realize new and/or better plasmonic behaviors $[3,4]$. In this paper we first use high-resolution transmission electron microscopy to present evidence for ferroplasmons, strong surface plasmons that appear in ferromagnetic metals when in the proximity of noble metal nanostructures. To better understand and explore the origin of these ferroplasmons we have developed a simple approach to synthesize electron transparent nanostructures so as to compare their plasmonic properties by electron energy loss spectroscopy and compare with calculations performed using the discrete dipole approximation (DDA) approach.

Fig. 1(a) shows the high angle annular dark field (HAADF) image of a Co-Ag bimetallic nanoparticle formed on an amorphous carbon substrate by pulsed laser dewetting. A Zeiss Libra 200 TEM with a monochromator and energy filter was used to characterize the nanostructure by HAADF mode and electron energy-loss spectroscopy (EELS) at $200 \mathrm{kV}$. To understand the spatial nature of surface plasmons in these nanoparticles we generated the maps of plasmons from different energy windows, with the 0 to $3 \mathrm{eV}$ data shown in Fig. 1(b-c) and 3 to $5 \mathrm{eV}$ data shown in Fig. 1(d-e). For the $0-3 \mathrm{eV}$ region, the strongest surface plasmon occurred at $2.6 \pm 0.3 \mathrm{eV}$ and measurement of the scattering probability [Fig. 1(b)] and full width half maxima (FWHM) [Fig. 1(c)] showed that the Co region had a strong plasmon, called the ferroplasmon. In contrast, analysis of the 3 to $5 \mathrm{eV}$ window showed that a surface plasmon occurred at energy of $3.5 \pm 0.3 \mathrm{eV}$ and the scattering probability is shown in Fig. 1(d), while the FWHM is shown in Fig. 1(e). This peak was seen to be highly localized to the Ag region. Similar measurements done on pure Co nanoparticles did not show any evidence for these strong ferroplasmons seen when Co was in the vicinity of Ag.

DDA modeling has suggested that ferroplasmons can be a result of electromagnetic coupling between the two metals [2]. To investigate this further, we developed a technique to rapidly prepare electron transparent nanostructures and perform EELS on at different energy windows and compare these experiments with DDA electrodynamics theory. The sample preparation involved a combination of nanosphere lithography and a carbon substrate float-off process. Fig. 1(f) shows the Z-contrast from HAADF imaging of pairs of non-touching bimetallic Co-Ag triangles 
synthesized by this technique on a-C. Fig. 1(g) shows the EELS spatial map of the scattering intensities in the window of 3.0 to $4.6 \mathrm{eV}$ for Fig. 1(f). Fig. 1(h) is the theoretical prediction of scatter- ing intensity from DDA simulation at an energy of $4.26 \mathrm{eV}$. Good agreement between theory and experiment was evident, with large scattering coming primarily from Ag for this energy regime.

The authors acknowledge support by the Army Research Office through grant W911NF-13- 10428. We also acknowledge CNMS2013-284 at the Center for Nanophase Materials Science, which is sponsored at ORNL by the Scientific User Facilities Division, Office of Basic Energy Sciences, U.S. Department of Energy for SEM characterization.

(a)

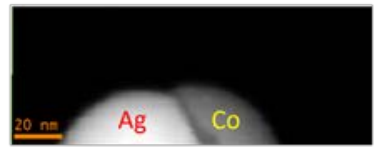

(b)

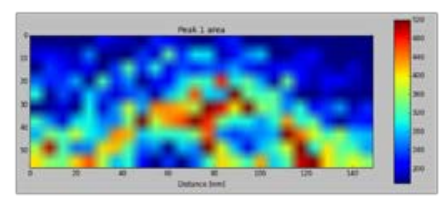

(d)

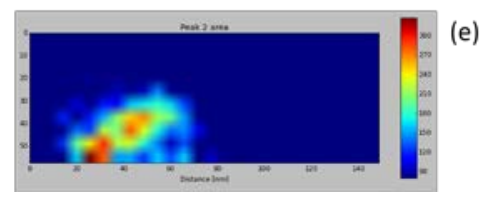

(C)

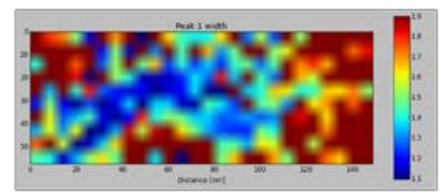

(e)

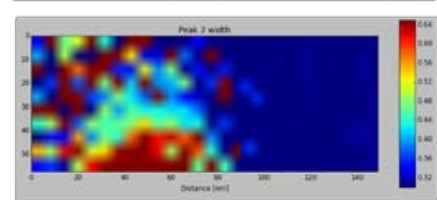

$(f)$

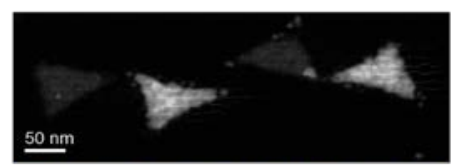

(g)

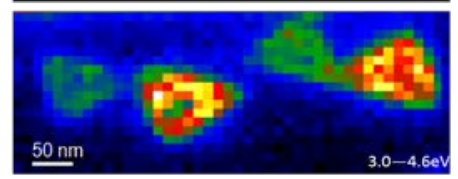

(h)

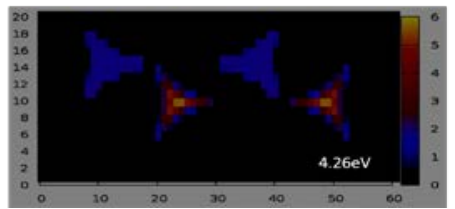

Figure 1: (a) HAADF of a Ag-Co bimetallic nanoparticle. The individual metallic regions are marked in the figure. (b) Scattering probability of the $2.6 \pm 0.3 \mathrm{eV}$ plasmon in ppm. (c) The FWHM of the $2.6 \pm 0.3 \mathrm{eV}$ plasmon in $\mathrm{eV}$. (d) Scattering probability of the $3.5 \pm 0.3 \mathrm{eV}$ plasmon in ppm. (e) The FWHM of the $3.5 \pm 0.3 \mathrm{eV}$ plasmon in $\mathrm{eV}$. (f) HAADF of Ag-Co triangle pairs. The brighter triangles are Ag while the darker triangles are Co. (g) EELS map between 3 to $4.6 \mathrm{eV}$ for the triangles in (f). (h) DDA simulation of the EELS from the triangles in (h) at 4.26 $\mathrm{eV}$.

\section{References}

[1] A. N. Grigorenko, A. K. Geim, H. F. Gleeson, Y. Zhang, A. A. Firsov, I. Y. Khrushchev, and J. Petrovic, "Nanofabricated media with negative permeability at visible frequencies," Nature, vol. 438, pp. 335-338, 2005.

[2] N. Passarelli, L. A. Perez, and E. A. Coronado, "Plasmonic interactions: From molecular plasmonics and fano resonances to ferroplasmons,” ACS Nano, vol. 8, no. 10, pp. 9723-9728, 2014. http://dx.doi.org/10.1021/nn505145v.

[3] R. Sachan, V. Ramos, A. Malasi, B. Bartley, G. Duscher, and R. Kalyanaraman, "Oxidation resistant ag nanoparticles for ultrastable plasmonic applications,” Adv. Mater., vol. 25, pp. 2045-2050, 2013.

[4] R. Sachan, A. Malasi, J. Ge, S. Yadavali, H. Krishna, A. Gangopadhyay, H. Garcia, G. Duscher, and R. Kalyanaraman, "Ferroplasmons: Intense localized surface plasmons in metal-ferromagnetic nanoparticles,” ACS Nano, vol. 8, pp. 9790-9798, 2014. 